\title{
Improvement of Idiopathic Cardiomyopathy After Colon Clear
}

\author{
Randa M. Nasrat ${ }^{\mathrm{a}}$, Mohammad M. Nasrat ${ }^{\mathrm{a}}$, Abdullah M. Nasrat ${ }^{\mathrm{b}, \mathrm{d}}$, Salwa A. Nasrat ${ }^{\mathrm{c}}$
}

\begin{abstract}
Background: The study aimed to illustrate the effect of colon clear on idiopathic myocardial dysfunction. Helicobacter pylori colonized the stomach since an immemorial time, as if both the stomach and the bacterium used to live together in peace harmless to each other. H. pylori could migrate or get forced to migrate to the colon; antibiotics are seldom effective against extra-gastric $H$. pylori strains. The association of $H$. pylori and some cardiovascular diseases like myocarditis and cardiomyopaty has been sufficiently mentioned in literature. The role played by the increased mucosal production of inflammatory mediators (cytokines) induced by $H$. pylori among patients with ischemic heart diseases has been also clearly illustrated. The clinical association of gastritis and carditis is controversial. Active lymphocytic myocarditis manifested by intractable ventricular tachycardia, non-specific intra-ventricular block, and myocardial dysfunction has been described in a young woman infected with $H$. pylori; an immune influence has been emphasized in that patient as a possible etiology behind the development of autoimmune myocarditis. It has been reported also in literature that a possible role of autoimmunity induced by $H$. pylori in cardiomyopathy cannot be excluded.
\end{abstract}

Methods: Three female patients with frank history of $H$. pylori dyspepsia and an age range of $41-47$ years have developed myocaditis complicated with cardiomyopathy as confirmed by echocardiography (ECG) and magnetic resonance imaging (MRI). Existence of H. pylori in the colon was confirmed by $H$. pylori fecal antigen. Colon clear was done for them.

Results: Symptomatic improvement and clinical recovery to sinus rhythm with minimal supra-ventricular extra-systoles occurred for all patients after colon clear. Patients continued improvement to normal cardiac tracing and normal left ventricular ejection function within

Manuscript accepted for publication April 08, 2015

aDepartment of Internal Medicine, Helwan Gen Hosp, Cairo, Egypt

${ }^{b}$ Department of Surgery, Balghsoon Clinics, Jeddah, KSA

'Department of Physiotherapy, Cardiac Surgery Academy, Cairo, Egypt

${ }^{\mathrm{d} C}$ Corresponding Author: Abdullah M. Nasrat, Department of Surgery, Balgh-

soon Clinics, Jeddah, KSA. Email: abdullahalnasrat@yahoo.com

doi: http://dx.doi.org/10.14740/cr398e further 3 - 4 weeks.

Conclusion: Colon clear could be a simple and safe measure to improve changes in cardiac rhythm, heart rate and myocardial function developing in association with $H$. pylori due to inflammatory, toxic or immune reasons.

Keywords: Cardiomyopathy; Helicobacter pylori; Myocarditis; Senna purge

\section{Introduction}

The widespread prevalence and the medical challenges constituted by Helicobacter pylori, namely its close relation to acid peptic disease, gastric carcinoma and lymphoma have led to the widely established medical concept that $H$. pylori eradication should be a necessary attempt $[1,2]$. H. pylori colonized the stomach since an immemorial time [2], as if both the stomach and the bacterium used to live together in peace harmless to each other. $H$. pylori could migrate or get forced to migrate to the colon due to the influence of antibiotic violence [2-4]; antibiotics are seldom effective against extra-gastric $H$. pylori strains [5].

The latest reports in literature demonstrate a definite flare up of many medical challenges related to $H$. pylori through immune or different unknown reasons [6-9]. The association of H. pylori and some cardiovascular diseases like myocarditis and cardiomyopaty has been sufficiently mentioned in literature. The role played by the increased mucosal production of inflammatory mediators (cytokines) induced by $\mathrm{H}$. pylori among patients with ischemic heart diseases has been also clearly illustrated [10-12].

The clinical association of gastritis and carditis is controversial; opinions are currently divided as whether it is the result of gastro-esophageal reflux or a proximal extension of H. pylori infection from the stomach [10]. Active lymphocytic myocarditis manifested by intractable ventricular tachycardia, non-specific intra-ventricular block, and myocardial dysfunction has been described in a young woman infected with $H$. pylori; an immune influence has been emphasized in that patient as a possible etiology behind the development of autoimmune myocarditis [11]. It has been also 


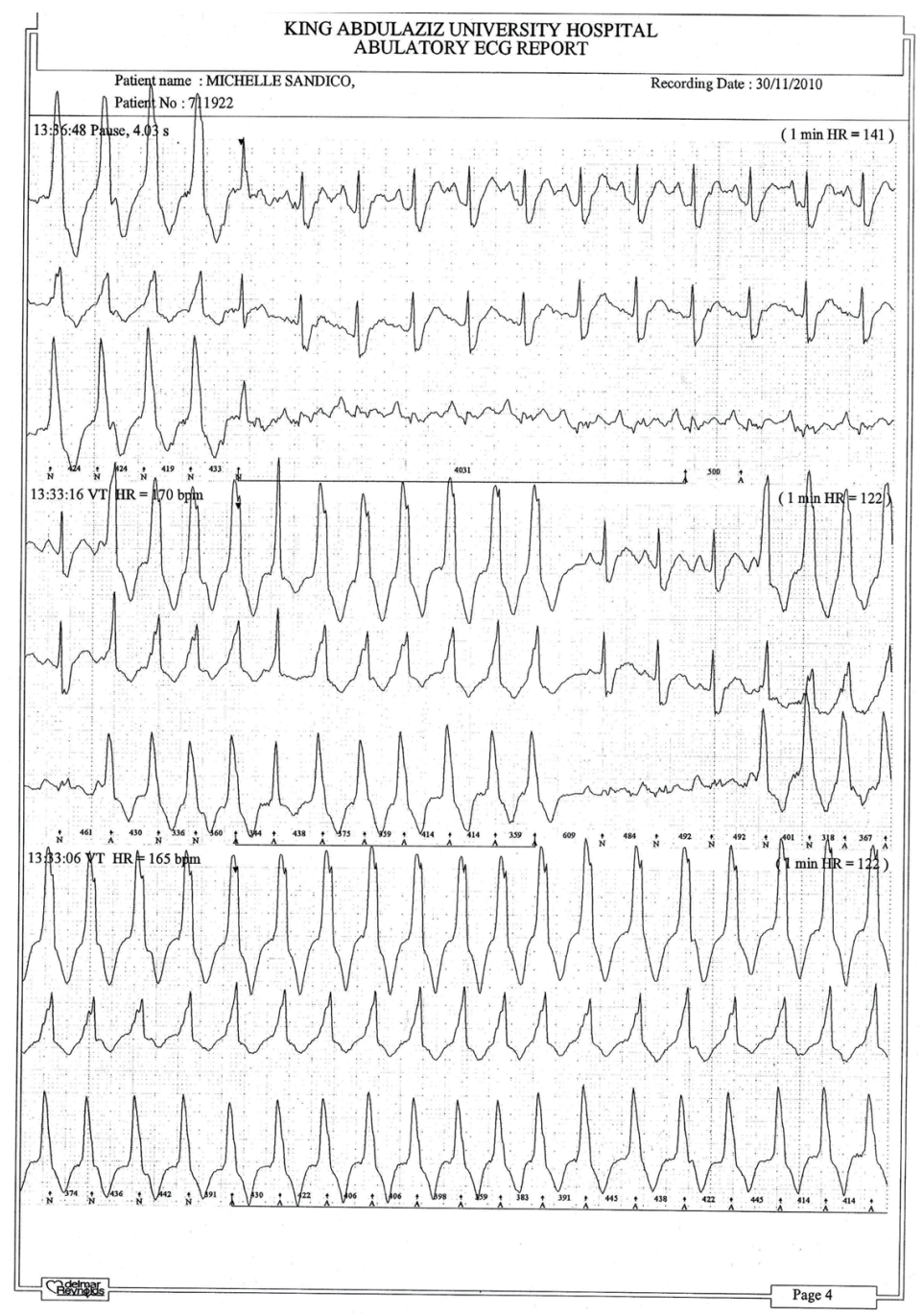

Figure 1. Part of the Holter monitor tracing of one patient before colon clear.

reported in literature that a possible role of autoimmunity induced by $H$. pylori in cardiomyopathy cannot be excluded [12]. Different reports in literature have confirmed the association of cytotoxin-associated gene A (cagA) positive $H$. pylori strains with many medical problems, and emphasized that cagA of $H$. pylori encodes a highly immunogenic and virulence-associated protein; the presence of this virulent gene in the body could affect the clinical outcome in many patients $[2,13]$.

The aim of this study was to illustrate the effect of colon clear on idiopathic myocardial dysfunction.

\section{Patients and Methods}

\section{Design and setting}

Multiple-case clinical study was done in Balghsoon Outpatient Clinics in Jeddah/Saudi Arabia during October 2012 - May
2013.

\section{Patients}

Three female patients with frank long history of $H$. pylori dyspepsia and an age range of $41-47$ years have developed palpitation, chest tightness and breathing discomfort with frequent extra-systoles in the echocardiography (ECG). Shortly, two of them developed ventricular extra-systoles; an initial diagnosis of viral myocaditis complicated with idiopathic cardiomyopathy was made based upon ECG and magnetic resonance imaging (MRI). Their left ventricular ejedtion fraction was ranging between $29 \%$ and $30 \%$. They were advised for insertion of an automated implantable cardioverter defibrillator (AICD) but they hesitated towards this procedure; therefore, they were put on medical treatment. Ventricular extra-systoles disappeared on medications but their distressing symptoms showed no improvement. H. pylori existence in the colon was confirmed by a reliable specific test, $H$. pylori fecal antigen 


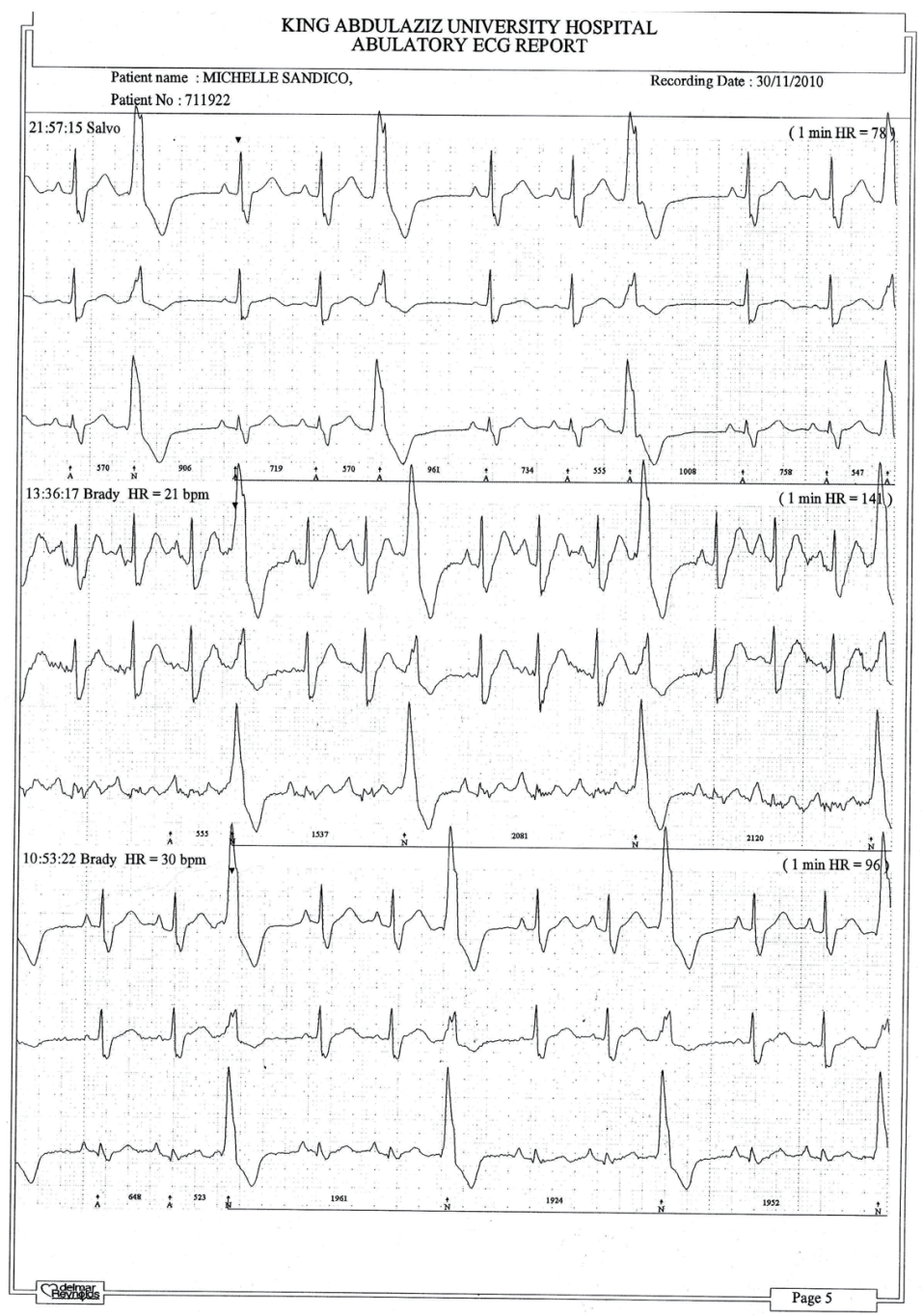

Figure 2. Another part of the Holter tracing of the same patient before colon clear.

[2]. Colon clear with the potent natural senna leaves purge was employed for them. Successful $H$. pylori eradication was confirmed by $H$. pylori fecal antigen test $[2,4]$. H. pylori fecal antigen test was available from Acon Laboratory, USA, Batch No. HP8040008.

\section{Ethical considerations}

An informed signed consent was taken from all patients, and they were free to quit the study whenever they like. All patients were allowed to follow their usual diet, medications and to lead their routine style of life. They were not requested to stop their medications but they did that gradually by their own because of physical uneasiness upon intake of pills. One of them stopped her medications all of a sudden as she missed them while traveling by road; she gave the expression of suddenly feeling health and freedom. The research proposal was approved and the study followed the rules of the Research Ethics Committee of King Faisal Specialized Hospital and Re- search Center in Jeddah, Saudi Arabia.

\section{Results}

The three patients expressed immediate dramatic improvement after colon clear; they became free of any symptoms instantly after diarrhea was complete and they were able to exercise walking for continuous $1 \mathrm{~h}$ without fatigue or doing more than $400 \mathrm{~m}$ walk exercise in less than $6 \mathrm{~min}$. One patient expressed "I am back myself again" while another patient said "now I can count my pulse". Their ECG resumed a sinus rhythm with few supra-ventricular extra-systoles, around $7-9 / \mathrm{min}$. The left ventricular ejection fraction became $47-49 \%$. The three patients achieved complete recovery within 3 - 4 weeks; their left ventricular function improved to $53-55 \%$ and the ECG tracing became straight forward normal. Patients were not obliged to stop medications but later they did that by their own because of feeling uneasy with medications and they kept maintaining their condition stable even they were feeling better expressing 


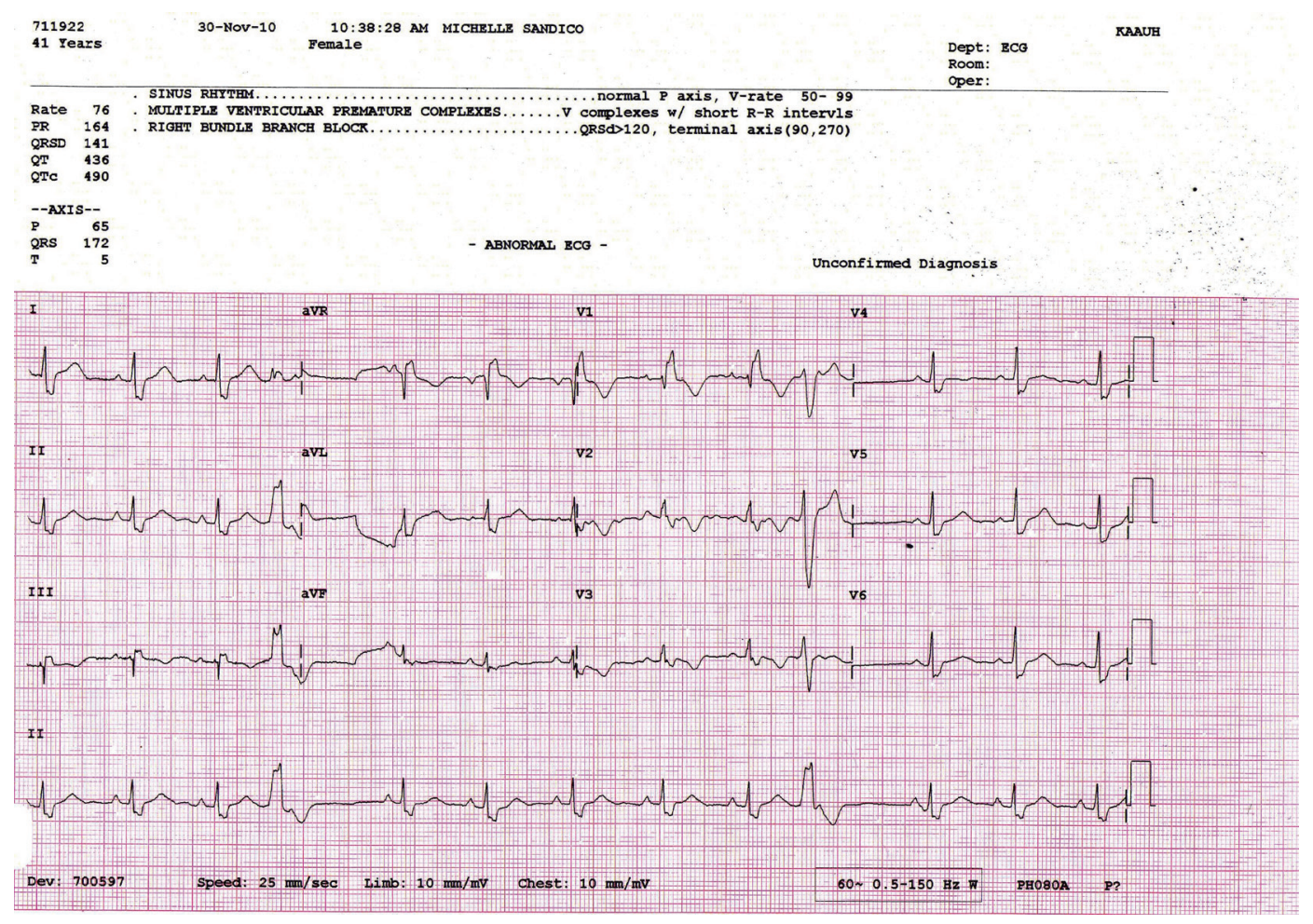

Figure 3. ECG tracing of the same patient before colon clear.

further physical relief upon quit of medications. Patients were followed up for 12 months showing no recurrence as they were watching carefully their colonic condition.

The results of this study were compared with the records of seven female patients of rather similar age range (40 - 49 years) and rather similar physical and clinical parameters who have developed myocarditis and cardiomypathy confirmed by ECG and MRI, they were put on the following several medications: amiodarone hydrochloride $200 \mathrm{mg}$ once daily, carvedilol $6.25 \mathrm{mg}$ once daily and candesartan cilexetil $8 \mathrm{mg}$ once daily together with two diuretic drugs (furosemide and spironolactone), a gstric sedative and aspirin in order to reduce symptoms and burden on the heart, while one patient was obliged to undergo AICD insertion because of risk on life. In spite of these measures, those patients were not getting much better as concerns palpitation, easy fatigability and chest discomfort.

The results of this study were further compared and confirmed also by the clinical study of one female patient aged 49.5 years who developed myocardititis and cardiomyopathy manifested with ventricular extra-systoles following a long history of $H$. pylori dyspepsia. She was advised for AICD insertion because of risk on her life but she was not able to afford the cost of this procedure, and she disappeared to reappear after 1 month symptom-less; she mentioned that she just followed camel milk intake with honey for 1 month. Her ECG was restored normal and her left ventricular ejection fraction improved from $31 \%$ to $49 \%$.

Figures 1 and 2 show parts of the Holter monitor tracing of one of the patients of the study before undergoing colon clear. Figure 3 shows the ECG tracing of the same patient before colon clear, while Figure 4 shows her ECG tracing after colon clear.

\section{Discussion}

Migration of $H$. pylori to the colon is a fact that has been reported in literature [2-4]. It was suggested that the antibiotic violence could have forced the stomach bacterium to migrate to the colon rather than eradicating it from the stomach $[2$, 4]. This suggestion is supported by the finding that pseudomembranous toxic colitis and toxic megacolon have developed after eradication of $H$. pylori from the stomach by antibiotic therapy $[14,15]$. H. pylori in the colon will continue producing ammonia for a reason or no reason leading to accumulation of profuse toxic amounts of ammonia, unopposed or buffered by any acidity. Ammonia is known to be toxic and colon spastic [16]; a colonic re-absorptive error could establish leading to excessive fluid and salt retention in the body with subsequent burden on blood pressure and heart rate. Accumulation of ammonia in the colon could lead to adverse toxic effects on the myocardium in susceptible people; toxic myocarditis and cardiomyopathy could be integral sequels of these toxic effects $[11,12]$.

The results of this study confirm the concept of improvement of cardiomyopathy after colon clear due to elimination of a potential source of toxins from the colon as the wonderful senna purge is potent and is known to effectively kill and/or expel the migrated colonic $H$. pylori strains [4, 17]. This concept is further supported by the results achieved by the patient 


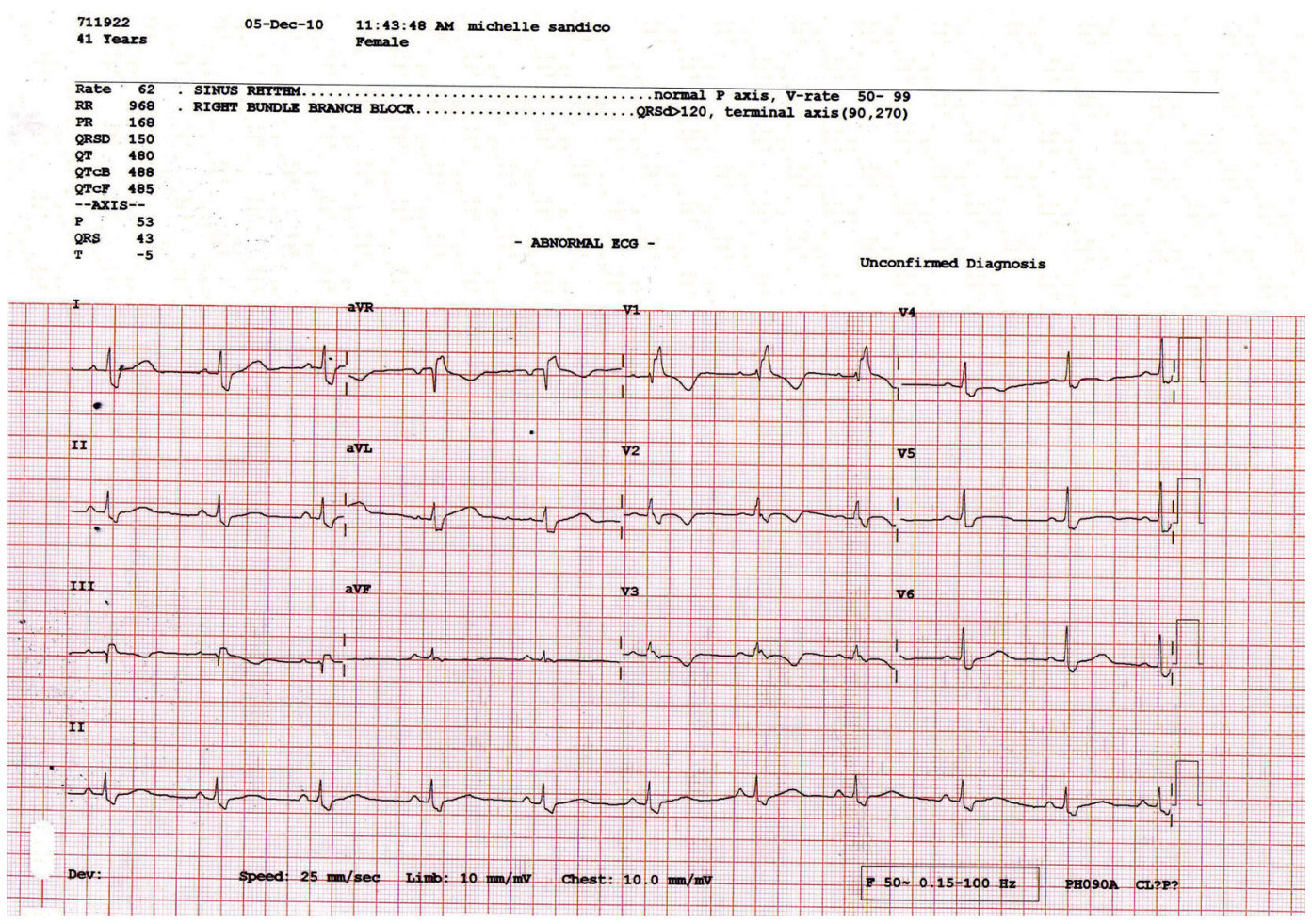

Figure 4. ECG tracing of the same patient after colon clear.

who followed the camel milk/honey therapeutic remedy; camel milk is a known potent colon clear measure while acetate which is directly lethal to $H$. pylori is existing among the end products of glucose utilization by the body [17-19].

In this study, the effect of the senna leaves extract on the growth of $H$. pylori was studied; addition of three times dilution of the senna leaves purge preparation to solid culture of $H$. pylori was found directly lethal to it.

The records of the seven female patients who followed medical treatment and AICD insertion in one of them failed to provide information about any history of $H$. pylori for those patients although it was expected as existence of abnormal $H$. pylori strains is common particularly in developing countries $[2,4,17]$, and because of their failure to respond to adequate medications, most probably due to persistence of an underlying etiologic pathology.

Toxic myocarditis rather than viral myocarditis could have been the pathological etiology behind many cases diagnosed as idiopathic cardiomyopathy during the last three decades. The value of this study is gained from the true promising opportunity that many cases of cardiomyopathy could be avoided and that many cases of the newly discovered condition could be cured or at least progress of the disease could be stopped.

A general impression has developed that those patients developing cardiomyopathy due to the influence of colonic $H$. pylori strains are disadvantaged susceptible individuals; they should watch their colonic condition and employ colon care and colon clear whenever they develop frank dyspeptic symptoms. It was also observed that females are more pre- disposed than males to these adverse toxic effects produced by the abnormal-behavior colonic $H$. pylori strains; therefore, further comparative studies concerning this regard including both male and female patients are recommended.

\section{Conclusion}

The senna leaves purge should be considered as a recognized potent colon clear measure. Toxic myocarditis rather than viral could be the pathologic etiology behind many cases of idiopathic cardiomyopathy; hence it could be prevented, the newly discovered conditions could be cured or at least progress of the disease could be stopped. Eradication of $H$. pylori from the colon via colon clear could lead to improvement of cardiomyopathy due to elimination of colonic $H$. pylori strains below its pathologic level. Colon clear could be a simple and safe measure to improve changes in cardiac rhythm, heart rate and myocardial function developing in association with $H$. pylori due to inflammatory, toxic or immune reasons.

\section{Acknowledgement}

The study appreciates the facilities and time allowed by Balghsoon Clinics in Jeddah, Saudi Arabia. The continuous encouragement of Abdul-Aziz Al-Sorayai Investment Company (ASIC) in Jeddah, Saudi Arabia and the scientific emotional support of Dr Ahmed S. Balghsoon are extremely valued and appreciated. 


\section{Conflict of Interest}

There is no conflict of interest existing.

\section{References}

1. Ge Z. Potential of fumarate reductase as a novel therapeutic target in Helicobacter pylori infection. Expert Opin Ther Targets. 2002;6(2):135-146.

2. Farinha P, Gascoyne RD. Helicobacter pylori and MALT lymphoma. Gastroenterology. 2005;128(6):1579-1605.

3. Matsuo S, Mizuta Y, Hayashi T, Susumu S, Tsutsumi R, Azuma T, Yamaguchi S. Mucosa-associated lymphoid tissue lymphoma of the transverse colon: a case report. World J Gastroenterol. 2006;12(34):5573-5576.

4. Nasrat AM. The world misconception and misbehavior towards Helicobacter pylori is leading to major spread of illness. The 7th Anti-Aging Medicine World Congress, Monte-Carlo, Monaco, 2009 Mar. Available from URL, www.euromedicom.com.

5. Grunberger B, Wohrer S, Streubel B, Formanek M, Petkov V, Puespoek A, Haefner M, et al. Antibiotic treatment is not effective in patients infected with Helicobacter pylori suffering from extragastric MALT lymphoma. J Clin Oncol. 2006;24(9):1370-1375.

6. Segni M, Borrelli O, Pucarelli I, Delle Fave G, Pasquino AM, Annibale B. Early manifestations of gastric autoimmunity in patients with juvenile autoimmune thyroid diseases. J Clin Endocrinol Metab. 2004;89(10):4944-4948.

7. Kountouras J, Zavos C, Chatzopoulos D. A concept on the role of Helicobacter pylori infection in autoimmune pancreatitis. J Cell Mol Med. 2005;9(1):196-207.

8. Kountouras J, Deretzi G, Zavos C, Karatzoglou P, Touloumis L, Nicolaides T, Chatzopoulos D, et al. Association between Helicobacter pylori infection and acute inflammatory demyelinating polyradiculoneuropathy. Eur J Neurol. 2005;12(2):139-143.

9. Veneri D, Krampera M, Franchini M. High prevalence of sustained remission of idiopathic thrombocytopenic purpura after Helicobacter pylori eradication: a long-term follow-up study. Platelets. 2005;16(2):117-119.

10. Owen DA. Gastritis and carditis. Mod Pathol. 2003;16(4):325-341.

11. Bilinska ZT, Grzybowski J, Szajewski T, Stepinska J, Michalak E, Walczak E, Wagner T, et al. Active lymphocytic myocarditis treated with murine OKT3 monoclonal antibody in a patient presenting with intractable ventricular tachycardia. Tex Heart Inst J. 2002;29(2):113-117.

12. Klausz G, Tiszai A, Lenart Z, Gyulai Z, Tiszlavicz L, Hogye M, Csanady M, et al. Helicobacter pylori-induced immunological responses in patients with duodenal ulcer and in patients with cardiomyopathies. Acta Microbiol Immunol Hung. 2004;51(3):311-320.

13. Pietroiusti A, Diomedi M, Silvestrini M, Cupini LM, Luzzi I, Gomez-Miguel MJ, Bergamaschi A, et al. Cytotoxin-associated gene-A--positive Helicobacter pylori strains are associated with atherosclerotic stroke. Circulation. 2002;106(5):580-584.

14. Kubo N, Kochi S, Ariyama I, Murata M, Furusyo N, Hayashi J. [Pseudomembranous colitis after Helicobacter pylori eradication therapy]. Kansenshogaku Zasshi. 2006;80(1):51-55.

15. Schweigart U, Franck H, Schepp W, Lehn N, Becker K, Classen M. [Toxic megacolon after Helicobacter pylori eradication therapy]. Internist (Berl). 1997;38(4):352354.

16. Andreoli TE. Cecil Essentials of Medicine. WB Saunders Company, 5th Ed: 2001; 334.

17. Nasrat SAM, Nasrat RM, Nasrat MM, et al. The dramatic spread of diabetes mellitus worldwide and influence of Helicobacter pylori. General Med. 2015;3(1):159. [Epub ahead of print].

18. Mendz GL, Hazell SL, Burns BP. Glucose utilization and lactate production by Helicobacter pylori. J Gen Microbiol. 1993;139(12):3023-3028.

19. Habib HM, Ibrahim WH, Schneider-Stock R, Hassan HM. Camel milk lactoferrin reduces the proliferation of colorectal cancer cells and exerts antioxidant and DNA damage inhibitory activities. Food Chem. 2013;141(1):148152. 